\title{
Influence of the free-electron concentration on the optical properties of zincblende GaN up to $1 \times 10^{20} \mathrm{~cm}^{-3}$
}

\author{
Elias Baron $\odot^{*}$ and Rüdiger Goldhahn \\ Institut für Physik, Otto-von-Guericke-Universität Magdeburg, Universitätsplatz 2, 39106 Magdeburg, Germany \\ Michael Deppe and Donat J. As \\ Department of Physics, University of Paderborn, Warburger Straße 100, 33098 Paderborn, Germany \\ Martin Feneberg \\ Institut für Physik, Otto-von-Guericke-Universität Magdeburg, Universitätsplatz, 2, 39106 Magdeburg, Germany
}

(Received 26 March 2019; revised manuscript received 30 August 2019; published 18 October 2019)

\begin{abstract}
We analyze the optical properties of zincblende gallium-nitride in the infrared and ultraviolet spectral range $(\approx 27 \mathrm{meV}-6.5 \mathrm{eV})$ experimentally by spectroscopic ellipsometry and provide a quantitative description of these results by $\mathbf{k} \cdot \mathbf{p}$ perturbation theory. Free-electron concentrations above $10^{20} \mathrm{~cm}^{-3}$ are achieved by introducing germanium as a donor. We determine the dielectric function as well as band filling effects like the Burstein-Moss shift and band gap renormalization. The Kane model for the band structure of semiconductors near the $\Gamma$-point allows to calculate the effective electron mass and to determine the nonparabolicity of the conduction band. At the same time, these results can be used to derive the free-electron concentration all-optically. The combination of Kane's model, Burstein-Moss shift, and band-gap renormalization can be used to expertly describe the measured transition energies up to $\approx 3.7 \mathrm{eV}$ dependent on the carrier concentration, yielding an averaged hole mass of $\approx 0.61 \mathrm{~m}_{\mathrm{e}}$ for the contributing valence bands.
\end{abstract}

DOI: 10.1103/PhysRevMaterials.3.104603

\section{INTRODUCTION}

Zincblende III-nitrides possess some properties that are believed to be advantageous over those of wurtzite nitrides, such as the absence of spontaneous and piezoelectric polarization [1,2]. However, zincblende nitrides are metastable [3], making their preparation more challenging than that of their wurtzite counterparts. Consequently, defect control is an important issue for cubic nitrides [4,5] and high defect densities are most likely a major obstacle preventing their usage in devices.

On the other hand, several breakthroughs concerning the control and quality of zincblende GaN [6-8] were reported very recently. For certain applications, like quantum-dotbased functionalities [2,9-19], a replacement of wurtzite by cubic nitrides seems possible in the near future. However, the band structure of zincblende GaN remains scarcely researched, despite the fact that, compared to wurtzite GaN, it is simpler due to its higher symmetry [20].

The knowledge of the exact shape of its conduction band is especially essential for understanding the technically most

\footnotetext{
*elias.baron@ovgu.de

Published by the American Physical Society under the terms of the Creative Commons Attribution 4.0 International license. Further distribution of this work must maintain attribution to the author(s) and the published article's title, journal citation, and DOI.
}

relevant highly $n$-type doped material [18,21-23]. Therefore, this study reports on the energy-dependent effective electron mass of zincblende GaN, i.e., sheds light onto its very significant nonparabolicity. To achieve this goal, degenerately doped GaN thin films are investigated, where the Fermi energy is pushed high into the conduction band. The highest electron concentrations are reached by substituting the standard donor silicon by germanium [24,25], similar to wurtzite GaN [26-29]. For carrier concentrations up and above $10^{20} \mathrm{~cm}^{-3}$, many-body interactions become increasingly important and must be considered correctly.

Here we perform optical investigations to study the plasmonic properties of highly doped cubic GaN and provide a quantitative description of its conduction band structure in the framework of Kane's model [30]. Considering band filling [31,32] and renormalization effects [33], our model also succeeds in describing the energy position of the interband absorption onset.

\section{EXPERIMENT}

For this study, cubic zincblende $\mathrm{GaN}$ (c-GaN) samples were grown by plasma-assisted molecular beam epitaxy (MBE). The c-GaN layer is deposited on a $3 \mathrm{C}-\mathrm{SiC} / \mathrm{Si}$ substrate and doped by $\mathrm{Ge}$ or $\mathrm{Si}$ [25]. One unintentionally doped sample is present for reference. A description of the different dopants in c-GaN can be found elsewhere [34-36]. Important properties of the samples like layer thicknesses, source 
TABLE I. Growth properties as measured by Hall-effect, secondary-ion mass spectrometry (SIMS) and reflectometric interference spectroscopy: $\mathrm{Ge}$ or $\mathrm{Si}$ effusion cell temperature $\left(T_{\mathrm{Ge}, \mathrm{Si}}\right)$, thickness of the c-GaN layer measured by SIMS $\left(d_{\text {SIMS }}\right)$ and reflection $\left(d_{\text {refl }}\right)$, free-carrier concentration measured by Hall-effect $\left(n_{\text {Hall }}\right)$ and corresponding Hall-mobility $\left(\mu_{\text {Hall }}\right)$. Sample D is the unintentionally doped reference.

\begin{tabular}{lcccccc}
\hline \hline Sample & Doping & $\begin{array}{c}T_{\mathrm{Ge}, \mathrm{Si}} \\
\left({ }^{\circ} \mathrm{C}\right)\end{array}$ & $\begin{array}{c}d_{\text {SIMS }} \\
(\mathrm{nm})\end{array}$ & $\begin{array}{c}d_{\text {refl }} \\
(\mathrm{nm})\end{array}$ & $\begin{array}{c}n_{\text {Hall }} \\
\left(\mathrm{cm}^{-3}\right)\end{array}$ & $\begin{array}{c}\mu_{\text {Hall }} \\
\left(\mathrm{cm}^{2} / \mathrm{Vs}\right)\end{array}$ \\
\hline $\mathrm{A}$ & $\mathrm{Ge}$ & 1000 & 365 & 363 & $3.7 \times 10^{20}$ & 63 \\
$\mathrm{~B}$ & $\mathrm{Ge}$ & 900 & 437 & 460 & $8.7 \times 10^{19}$ & 90 \\
$\mathrm{C}$ & $\mathrm{Ge}$ & 800 & 617 & 543 & $2.7 \times 10^{18}$ & 105 \\
$\mathrm{D}$ & - & - & 612 & 612 & - & - \\
$\mathrm{E}$ & $\mathrm{Si}$ & 1100 & - & 564 & $3.8 \times 10^{19}$ & 74 \\
$\mathrm{~F}$ & $\mathrm{Si}$ & 1050 & - & 611 & $7.6 \times 10^{18}$ & 77 \\
\hline \hline
\end{tabular}

temperatures, or results of Hall-measurements, are summarized in Table I.

The samples were investigated by spectroscopic ellipsometry on two different instruments. A Woollam IR-VASE based on Fourier-transform infrared spectroscopy was employed from 300-6000 $\mathrm{cm}^{-1}$, while a Woollam VASE (a scanning variable-angle spectroscopic ellipsometer on a grating monochromator) operated in the range from $0.5-6.5 \mathrm{eV}$. Both instruments have overlapping spectral ranges. The infrared spectroscopic ellipsometry measurements were performed with a resolution of $4 \mathrm{~cm}^{-1}$ while the ultraviolet measurements were done with a step size of $10 \mathrm{meV}$. Spectroscopic ellipsometry yields to the so-called ellipsometric angles $\Psi$ and $\Delta$, which yield the complex reflection coefficient $\rho$ [37]. All measurements were performed at different angles of incidence $\Phi$. In this study, $\Phi$ was chosen to be $50^{\circ}, 60^{\circ}$, and $70^{\circ}$, respectively. The reflection coefficient in combination with the angle of incidence can be used to determine a so-called pseudodielectric function $\langle\varepsilon\rangle$ :

$$
\begin{aligned}
\rho & =\frac{R_{\mathrm{p}}}{R_{\mathrm{s}}}=\tan (\Psi) e^{i \Delta} \\
\langle\varepsilon\rangle & =\sin ^{2}(\Phi)\left[1+\tan ^{2}(\Phi)\left(\frac{1-\rho}{1+\rho}\right)^{2}\right] .
\end{aligned}
$$

Here $R_{\mathrm{p}}$ and $R_{\mathrm{s}}$ are the Fresnel reflection coefficients for $p$ and $s$-polarized light.

The measured ellipsometric angles $\Psi$ and $\Delta$ were analyzed by constructing a multilayer model, which represents the layout of the samples consisting of $\mathrm{c}-\mathrm{GaN}, 3 \mathrm{C}-\mathrm{SiC}$, and $\mathrm{Si}$ from surface to substrate. For the infrared spectral range, the c-GaN layer was characterized by a model for the dielectric function (DF) as described in Sec. III A, while a general oscillator model was used to fit the line shape of the measured data in the visible and ultra-violet spectral region. The applied models were fitted to the experimental data ( $\Psi$ and $\Delta$ ) by numerically minimizing mean squared error values using a Levenberg-Marquardt algorithm. The resulting model DF was used as a starting point to perform a point-by-point (pbp) fit, which numerically changed the value of the DF wavelengthby-wavelength until the best agreement with the experimental data was achieved $[38,39]$. Then, the resulting pbp-DF was again line-shape fitted by the model described in Sec. III A for the IR to extract the characteristic parameters. In contrast, in the UV spectral range, the pbp-DF comprised our final result because we only needed the value of the transition energy which was derived as described below. A more detailed description of the analysis can be found elsewhere [40-42]. The final results of our spectroscopic ellipsometry analysis are DFs (model and pbp fitted) for the differently high doped c-GaN layers. The substrate was already thoroughly investigated earlier [43-47].

Additionally, Hall-, Raman, reflectometric interference spectroscopy, and secondary-ion mass spectrometry measurements were performed. For the Hall-effect measurements, the van der Pauw geometry was used. A 532-nm laser (output power $100 \mathrm{~mW}$ ) was used to perform the Raman measurements and was focused on the samples by a microscope. The signal was analyzed by a monochromator with $750-\mathrm{mm}$ focal length and a grating density of $2400 / \mathrm{mm}$, and was recorded by an attached charge coupled device camera.

\section{THEORY}

In this section, we introduce the model for the DF in the infrared spectral range, which describes contributions due to phonons and free carriers. Furthermore, a model description for the band structure is presented, which is valid in the vicinity of the $\Gamma$-point of the Brillouin zone, for example, close to the onset of the interband absorption.

\section{A. Infrared dielectric function}

The dielectric response of any system can be described by the complex DF

$$
\varepsilon(\omega)=\varepsilon_{1}(\omega)+i \varepsilon_{2}(\omega) .
$$

In the infrared region, for the DF, phonon and plasmon contributions have to be considered, which account for lattice oscillations and the response of free carriers, respectively [42]. In the simplest case, the phonon contribution can be described by the Lorentz model, which considers the electrons bound to the nuclei in much the same way as a small mass can be bound to a large mass by a spring. Harmonic oscillations arise. On the other hand, the plasmon contribution is described by the Drude model, which derives directly from the Lorentz model without a restoring force [48]. The infrared DF can then be written as:

$$
\varepsilon(\omega)=\varepsilon_{\infty}+\sum_{l} \frac{S_{l} \omega_{\mathrm{TO} l}^{2}}{\omega_{\mathrm{TO} l}^{2}-\omega^{2}-i \gamma_{\mathrm{TO} l} \omega}-\frac{\omega_{\mathrm{P}}^{2}}{\omega^{2}+i \gamma_{\mathrm{P}} \omega} .
$$

Here, $S_{l}$ is the amplitude, $\gamma_{\mathrm{TO} l}$ the broadening and $\omega_{\mathrm{TO}} l$ the resonance frequency of the $l$-th transverse optical (TO) phonon. $\varepsilon_{\infty}$ is the dielectric limit, representing contributions from higher energy oscillators and is reported to be 5.31 [49].

The plasma frequency $\omega_{\mathrm{P}}$ in the Drude term is of particular importance for the analysis of doped samples since $\omega_{\mathrm{P}}$ is dependent on the free-carrier concentration $n$ and the optical effective electron mass $m_{\mathrm{opt}}^{*}$, as seen in Eq. (4). $\gamma_{\mathrm{P}}$ is the 
plasma broadening:

$$
\omega_{\mathrm{P}}^{2}=\frac{e^{2} n}{\varepsilon_{0} m_{\mathrm{opt}}^{*}} .
$$

\section{B. Band structure model}

To express the nonparabolicity of the conduction band and, therefore, the dispersion, we use the model first introduced by Kane in 1956 [30], which is based on the $\mathbf{k} \cdot \mathbf{p}$ perturbation theory. We assume direct band gap at the zone center, isotropic bands and a scalar effective mass. Furthermore, we consider one conduction band and three valence bands, namely the heavy-hole, light-hole, and, due to spin-orbit interactions, the split-off valence band. The interaction between the conduction and the valence bands can be described by a $4 \times 4$ matrix, from which the following eigenvalue equation is derived:

$E^{\prime 3}+\left(\Delta_{\text {so }}-E_{\mathrm{G}}\right) E^{\prime 2}-\left(P^{2} k^{2}+E_{\mathrm{G}} \Delta_{\text {so }}\right) E^{\prime}-\frac{2}{3} P^{2} k^{2} \Delta_{\text {so }}=0$.

It should be noted that the dispersion $E(k)$ is given by:

$$
E(k)=E^{\prime}+\frac{\hbar^{2} k^{2}}{2 m_{\mathrm{e}}},
$$

where $m_{\mathrm{e}}$ is the free-electron mass. The spin-orbit splitting of the valence bands is described by $\Delta_{\text {so }}$ and the parameter $P$ is a momentum matrix element [30], which is assumed to be $k$-independent [50].

The third order equation in (5) can be analytically solved by Cardano's method for cubic equations. However, while an approximation for $\Delta_{\mathrm{so}} \gg E_{\mathrm{G}}$ is very popular [30]:

$$
E(k)=\frac{\hbar^{2} k^{2}}{2 m_{\mathrm{e}}}+\frac{1}{2}\left(E_{\mathrm{G}}+\sqrt{E_{\mathrm{G}}^{2}+\frac{8}{3} P^{2} k^{2}}\right),
$$

it does not apply to c-GaN, since $\Delta_{\text {so }}=15 \mathrm{meV}[49,51]$ and $E_{\mathrm{G}}=3.23 \mathrm{eV}[49,52]$ at room temperature. Instead, $\Delta_{\text {so }} \ll$ $E_{\mathrm{G}}$, which yields the following approximation for the conduction band dispersion [53]:

$$
E_{\mathrm{C}}(k)=\frac{\hbar^{2} k^{2}}{2 m_{\mathrm{e}}}+\frac{1}{2}\left(E_{\mathrm{G}}+\sqrt{E_{\mathrm{G}}^{2}+4 P^{2} k^{2}}\right) .
$$

This approximation is already used in different contexts, for example, by Wu et al. [53] for wurtzite InN. For further discussion, we define the $E_{\mathrm{P}}$ parameter which has the dimension of an energy [54]:

$$
E_{\mathrm{P}}=\frac{2 m_{\mathrm{e}}}{\hbar^{2}} P^{2}
$$

\section{Optical effective electron mass}

Previous computational investigations on the effective mass in zincblende $\mathrm{GaN}$ suggest that a single mass component represents the effective electron mass tensor sufficiently due to the direct band gap of cubic GaN [54-56]. We introduced the plasma frequency $\omega_{\mathrm{P}}$, which is dependent on the quotient $n / m_{\text {opt }}^{*}$, in the DF. The determination of the effective mass by analyzing the plasma frequency was already established [57-59]. We want to emphasize, that $m_{\mathrm{opt}}^{*}$ in Eq. (4) is the optical effective mass of the electrons in the conduction band averaging over all electrons in the conduction band. To calculate the value of the optical mass, we need to analyze the integral form of the quotient $n / m_{\text {opt }}^{*}$, which is given in Eq. (12) $[60,61]$. This is necessary since plasma oscillations affect electrons in a $k$ space determined by the Fermi-Dirac distribution [61]. A similar approach was published recently by Whalley et al. [23]. The equation originates from the definition of the carrier concentration $n$ and consists of the density of states $D(k)$ for electrons in the conduction band, the Fermi-Dirac distribution $f_{\mathrm{e}}$, the effective electron mass $m^{*}(k)[23,60,62,63]$ :

$$
\begin{gathered}
m^{*}(k)=\frac{\hbar^{2} k}{\partial E_{\mathrm{C}} / \partial k}, \\
\frac{1}{m^{*}(k)}=\frac{1}{m_{\mathrm{e}}}+\frac{2 P^{2}}{\hbar^{2} \sqrt{E_{\mathrm{G}}^{2}+4 P^{2} k^{2}}},
\end{gathered}
$$

and the crystal volume $V$. Approximating $f_{\mathrm{e}}$ by a step function (strictly valid at $T=0 \mathrm{~K}$ ) we obtain:

$$
\begin{aligned}
\frac{\omega_{\mathrm{P}}^{2} \varepsilon_{0}}{e^{2}} & =\frac{n}{m_{\mathrm{opt}}^{*}}=\int_{0}^{\infty} \frac{D(k) f_{\mathrm{e}}}{V m^{*}(k)} d^{3} k=\frac{1}{\pi^{2}} \int_{0}^{k_{\mathrm{F}}} \frac{k^{2}}{m^{*}(k)} d k \\
& =\frac{1}{\hbar^{2} \pi^{2}} \int_{0}^{k_{\mathrm{F}}} k \frac{\partial E_{\mathrm{C}}}{\partial k} d k
\end{aligned}
$$

with the Fermi-vector $k_{\mathrm{F}}=\left(3 \pi^{2} n\right)^{\frac{1}{3}}$. For $E_{\mathrm{C}}(k)$ we substitute the approximation given in Eq. (8). The result can be written as:

$$
\begin{aligned}
\frac{n}{m_{\mathrm{opt}}^{*}}= & \frac{1}{\hbar^{2} \pi^{2}}\left[\frac{\hbar^{2} k_{\mathrm{F}}^{3}}{3 m_{\mathrm{e}}}+\frac{1}{4} k_{\mathrm{F}} \sqrt{E_{\mathrm{G}}^{2}+4 P^{2} k_{\mathrm{F}}^{2}}\right. \\
& \left.-\frac{E_{\mathrm{G}}^{2}}{8 P} \ln \left(\frac{2 P k_{\mathrm{F}}+\sqrt{E_{\mathrm{G}}^{2}+4 P^{2} k_{\mathrm{F}}^{2}}}{E_{\mathrm{G}}}\right)\right] .
\end{aligned}
$$

This analytical expression only depends on the parameter $P$ (which is proportional to $\sqrt{E_{\mathrm{P}}}$ ), the free-carrier concentration $n$, and the band gap $E_{\mathrm{G}}$ as a material constant. Furthermore, if the carrier concentration is close to zero, the optical effective mass must match the effective mass at the $\Gamma$-point of the Brillouin zone for the undoped material [band mass $m_{0}^{*}(\Gamma)$ ] and is then only dependent on the band-gap energy $E_{\mathrm{G}}$ and $P$. This means that, if the band mass and the band gap of a material are established by other means, we are able to determine $P$ of that particular material by simply varying its value until the correct band mass is achieved. The same argument can be made for the effective mass at the Fermivector [see Eq. (10), $m^{*}\left(k=k_{\mathrm{F}}\right)$ ] and yields the same results for the value of $P$. Furthermore, to verify this method, the same result can be achieved by using the equation:

$$
E_{\mathrm{P}}=E_{\mathrm{G}}\left(\frac{m_{\mathrm{e}}}{m_{0}^{*}(\Gamma)}-1\right),
$$

which derives from Eq. (11) for $k=0$.

\section{Many-body effects}

In degenerately doped materials, many-body interactions play an important role in determining interband transition 


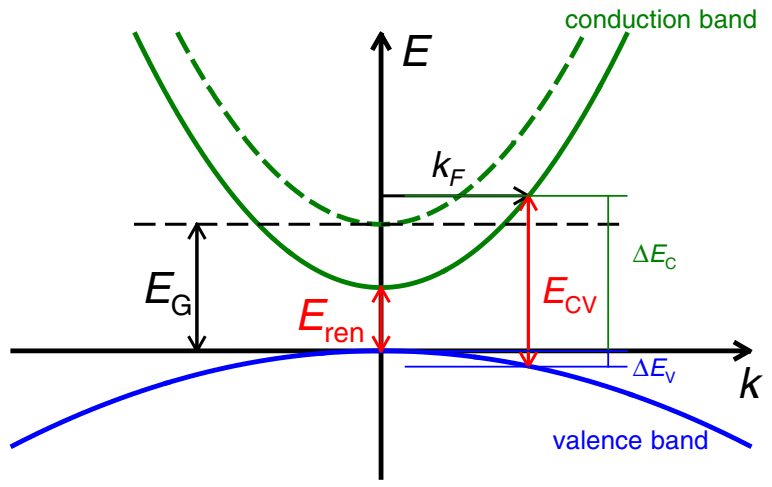

FIG. 1. Schematic description of the change in dispersion from an undoped (dashed) to degenerately doped (continuous) material. Increasing free-electron concentration leads to a decrease of the band gap $E_{\mathrm{G}}$ to $E_{\mathrm{ren}}$. Furthermore, the Fermi-vector $k_{\mathrm{F}}$ and therefore the transition energy $E_{\mathrm{CV}}$, consisting of conduction $\left(\Delta E_{\mathrm{C}}\right)$ and valence $\left(\Delta E_{\mathrm{V}}\right)$ band contributions, increases.

energies since they cause a change of the band structure, see Fig. 1. One of those many-body interactions is the so-called band-gap renormalization (BGR) [33,53], which describes a decrease of the band gap due to electron-electron $\left[\Delta E_{\mathrm{ee}}(n)\right]$ and electron-ion $\left[\Delta E_{\mathrm{ei}}(n)\right]$ interactions. The value of the BGR $\left[\Delta E_{\mathrm{BGR}}(n)\right]$ is the sum of both contributions [42,64], yielding the renormalized band gap $E_{\text {ren }}$ :

$$
\begin{aligned}
E_{\mathrm{ren}}(n) & =E_{\mathrm{G}}-\Delta E_{\mathrm{BGR}}(n) \\
& =E_{\mathrm{G}}-\Delta E_{\mathrm{ee}}(n)-\Delta E_{\mathrm{ei}}(n) .
\end{aligned}
$$

The interaction contributions are analytically approximated by [33]:

$$
\begin{gathered}
\Delta E_{\mathrm{ee}}(n)=\frac{e^{2} k_{\mathrm{F}}}{2 \pi^{2} \varepsilon_{0} \varepsilon_{\mathrm{s}}}+\frac{e^{2} k_{\mathrm{TF}}}{8 \pi \varepsilon_{0} \varepsilon_{\mathrm{s}}}\left[1-\frac{4}{\pi} \arctan \left(\frac{k_{\mathrm{F}}}{k_{\mathrm{TF}}}\right)\right], \\
\Delta E_{\mathrm{ei}}(n)=\frac{e^{2} n}{\varepsilon_{0} \varepsilon_{\mathrm{s}} a_{\mathrm{B}} k_{\mathrm{TF}}^{3}} .
\end{gathered}
$$

Here, $k_{\mathrm{TF}}$ is the Thomas-Fermi screening vector, $a_{\mathrm{B}}$ the effective Bohr-radius [both given in Eq. (18)] and $\varepsilon_{\mathrm{s}}=$ $\lim _{\omega \rightarrow 0}\left(\varepsilon_{1}\right)$ a static dielectric constant depending on the material. For c-GaN, this value is reported to be $\varepsilon_{\mathrm{s}}=9.44$ [49]:

$$
k_{\mathrm{TF}}=\sqrt{\frac{4 k_{\mathrm{F}}}{\pi a_{\mathrm{B}}}}, \quad a_{\mathrm{B}}=\frac{4 \pi \varepsilon_{0} \varepsilon_{\mathrm{s}} \hbar^{2}}{m^{*} e^{2}} .
$$

It has to be noted, that the calculation of $a_{\mathrm{B}}$ in Eq. (18) requires an effective electron mass $m^{*}$. This leads to a recursive function for the optical electron mass. To circumvent this problem, we self-consistently (iteratively) solve for $m_{\mathrm{opt}}^{*}$, starting from the effective electron mass at the $\Gamma$-point of the Brillouin zone. Already after few iterations no further changes of the BGR or the optical electron mass can be observed.

On the other hand, the Fermi energy and vector are increasing as a function of the carrier concentration due to the phase-space filling of the conduction band by electrons. This yields an increasing transition energy for the optical absorption onset between the valence and the conduction band. This effect is called the Burstein-Moss shift (BMS) [31,32]. It consists of conduction band $\left(\Delta E_{\mathrm{C}}\right)$ and valence band $\left(\Delta E_{\mathrm{V}}\right)$ contributions, which are represented by the dispersions of the bands:

$$
E_{\mathrm{BMS}}=\Delta E_{\mathrm{C}}+\Delta E_{\mathrm{V}}=E_{\mathrm{C}}\left(k_{\mathrm{F}}\right)+\left|E_{\mathrm{V}}\left(k_{\mathrm{F}}\right)\right| .
$$

The conduction band contribution is already expressed by Eq. (8). In regard to the valence bands, we approximately describe them by averaging over the three topmost bands (light hole, heavy hole, and split-off bands) by using an averaged effective hole mass $\bar{m}_{\mathrm{h}}$ in a parabolic expression:

$$
E_{\mathrm{V}}(k)=-\frac{\hbar^{2} k^{2}}{2 \bar{m}_{\mathrm{h}}} .
$$

A more detailed approach for the valence band contribution would be to use, e.g., the Luttinger-Kohn model [65] to determine the valence band dispersion of all valence bands and then calculate the energies at the Fermi-vector. However, here we are not able to experimentally distinguish between contributions from different valence bands, thus we omit the more complicated Luttinger-Kohn approach. Consequently, the averaged effective hole mass is empirically found to be $\bar{m}_{\mathrm{h}}=0.61 \mathrm{~m}_{\mathrm{e}}$ as described below.

Finally, to describe the experimentally observed transition energy as a function of the carrier concentration, we consider the BGR in the band dispersion. Therefore, we replace $E_{\mathrm{G}}$ by $E_{\text {ren }}$ in Eq. (8) yielding an interband transition energy of:

$$
E_{\mathrm{CV}}=\frac{\hbar^{2} k_{\mathrm{F}}^{2}}{2}\left(\frac{1}{m_{e}}+\frac{1}{\bar{m}_{\mathrm{h}}}\right)+\frac{1}{2}\left(E_{\mathrm{ren}}+\sqrt{E_{\mathrm{ren}}^{2}+4 P^{2} k_{\mathrm{F}}^{2}}\right) .
$$

\section{RESULTS AND DISCUSSION}

\section{A. Infrared spectroscopic ellipsometry}

For each sample, infrared spectroscopic ellipsometry (IRSE) data are analyzed by the model described in Sec. III A. As an example, we present ellipsometric angles and the pbp fit of sample B with $n_{\text {Hall }}=8.7 \times 10^{19} \mathrm{~cm}^{-3}$ in Fig. 2 . An excellent agreement for all three angles of incidence is achieved. Above $\approx 1000 \mathrm{~cm}^{-1}$, the spectrum is dominated by Fabry-Pérot oscillations due to the different layer thicknesses. Furthermore, the resulting DF of this sample is presented in Fig. 3.

Inspection of the real and imaginary parts of the DF yields a phonon contribution, visible as a peak in $\varepsilon_{2}$ and as oscillator structure in $\varepsilon_{1}$ at $\approx 550 \mathrm{~cm}^{-1}$. Additionally, the imaginary part increases to lower wave numbers, while the real part decreases. This behavior is due to the Drude contribution.

In detail, we find that the pbp-DF for $\varepsilon_{1}$ and $\varepsilon_{2}$ has to be described properly by a model DF with a phonon contribution consisting of two TO phonons. The dominant one is found at $\omega_{\mathrm{TO} 1}=(552 \pm 0.4) \mathrm{cm}^{-1}$ and an additional weaker structure at $\omega_{\mathrm{TO} 2}=(544 \pm 2) \mathrm{cm}^{-1}$. This apparent split of the TO phonon in c-GaN has been observed before [66-68] at the same phonon energy positions (within $\pm 2 \mathrm{~cm}^{-1}$ ) and is explained by the occurrence of anharmonic effects [69]. Extracted parameters for the DF are given in Table II. 


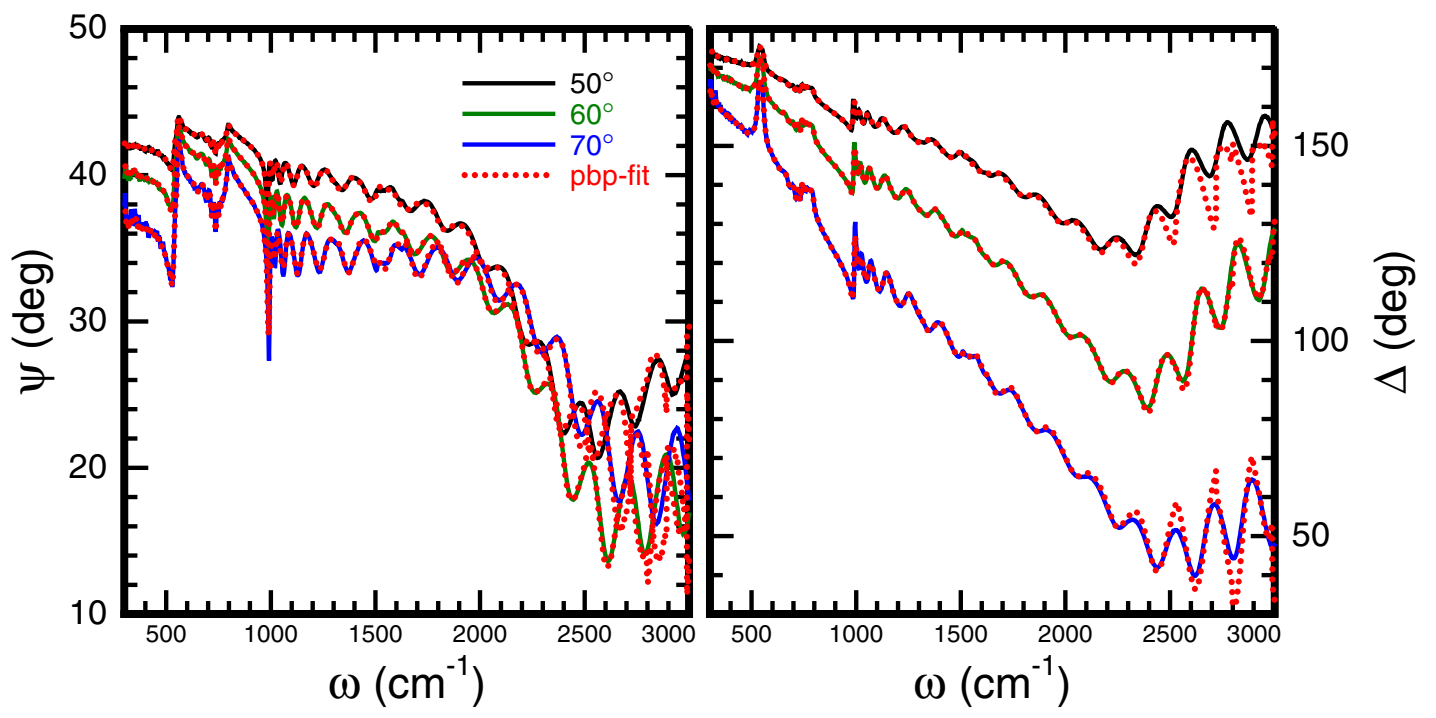

FIG. 2. Ellipsometric parameters $\Psi$ and $\Delta$ of sample $\mathrm{B}\left(n_{\text {Hall }}=8.7 \times 10^{19} \mathrm{~cm}^{-3}\right)$ in the infrared range measured by spectroscopic ellipsometry for three angles of incidence (black, green, blue, continuous curves) and the corresponding point-by-point-fit (pbp-fit, red, dotted curves). The feature at $\approx 1000 \mathrm{~cm}^{-1}$ can be attributed to the $3 \mathrm{C}$-SiC substrate, while Fabry-Pérot oscillations are visible for higher wave numbers.

For comparison, the ellipsometric parameters $\Psi$ and $\Delta$ as well as the real and imaginary parts of the DF of the unintentionally doped sample $\mathrm{D}$ are shown in Figs. 4 and 5. The change in the spectra and the DF are very pronounced, especially the absence of the plasmon contribution in $\varepsilon_{2}$. The splitting of the TO phonon remains present.

The DFs for $\varepsilon_{2}$ of all samples are shown in Fig. 6. The increasing effect of the plasmon contribution with increasing carrier concentration is clearly visible, while the TO phonon frequencies remain unchanged.
After the infrared DFs have been determined, the plasma frequencies $\omega_{\mathrm{P}}$ are extracted as parameters from the analytical DF (see Table II) and can be used for the analysis introduced in Sec. IIIC. The optical effective electron mass is a function of the carrier concentration [Eqs. (12) and (13)]. At $n \rightarrow 0$, the effective electron mass at the $\Gamma$-point of the Brillouin zone $(k=0)$ has to be obtained by our model as described in Sec. IIIC. Different references suggest $E_{\mathrm{G}} \approx$ $3.23 \mathrm{eV}[49,51,70]$ as experimental results and $m^{*}(k=0)=$ $m_{0}^{*} \approx 0.19 \mathrm{~m}_{\mathrm{e}}[54,56,71]$ from computational methods. For further analyses, we use $E_{\mathrm{G}}=3.23 \mathrm{eV}$ and $m_{0}^{*}=0.19 \mathrm{~m}_{\mathrm{e}}$

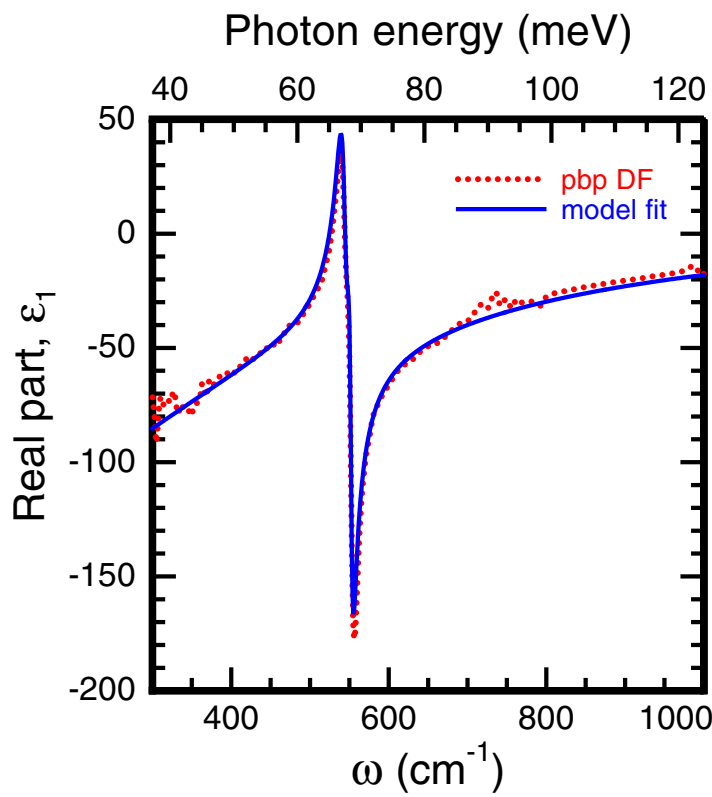

Photon energy ( $\mathrm{meV}$ )

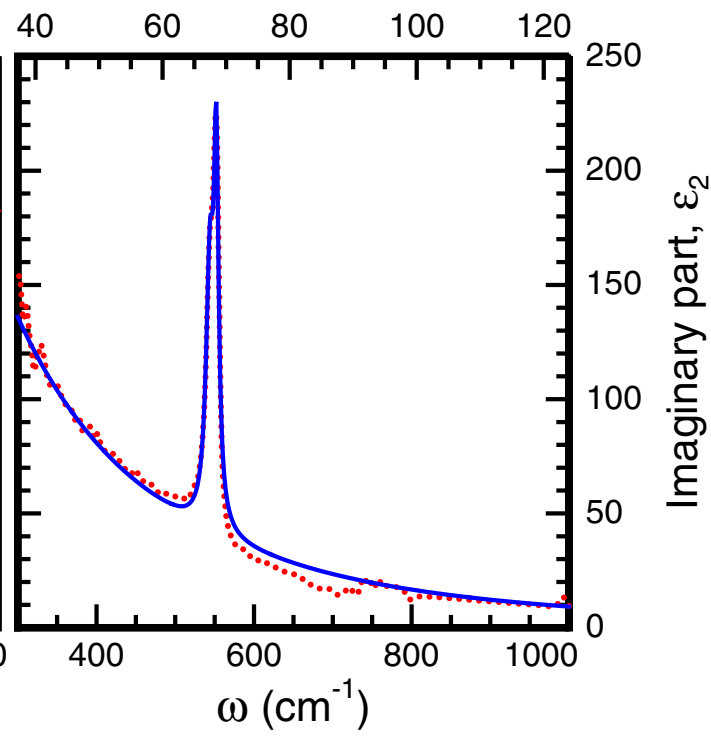

FIG. 3. Point-by-point fitted (pbp DF, dotted curve) and analytical model (model fit, continuous curve) dielectric function for sample B $\left(n_{\text {Hall }}=8.7 \times 10^{19} \mathrm{~cm}^{-3}\right)$ in the infrared range. 
TABLE II. Characterization results of the investigated samples as measured by infrared and ultraviolet spectroscopic ellipsometry: Thickness of the c-GaN $\left(d_{\text {UVSE }}\right)$ and the surface roughness layer $\left(d_{\text {rough }}\right)$, parameters of the dielectric function as described in Sec. III A as well as the measured $\left(E_{\mathrm{CV}, \mathrm{UVSE}}\right)$ and calculated transition energy $\left(E_{\mathrm{CV}}\right)$. The plasma frequency $\omega_{\mathrm{P}}$ yields the carrier concentration $n_{\mathrm{IRSE}}$ and the optical effective electron mass $m_{\mathrm{opt}}^{*}$ as described in Sec. IIIC for an estimated band mass of $m^{*}(k=0)=m_{0}^{*}=0.19 \mathrm{~m}_{\mathrm{e}}$ and therefore $E_{\mathrm{P}}=13.77 \mathrm{eV} . n_{\mathrm{Hall}}$ is again the free-carrier concentration measured by Hall effect.

\begin{tabular}{|c|c|c|c|c|c|c|c|c|c|c|c|c|c|c|c|}
\hline Sample & $\begin{array}{c}n_{\text {Hall }} \\
\left(\mathrm{cm}^{-3}\right)\end{array}$ & $\begin{array}{c}d_{\text {UVSE }} \\
(\mathrm{nm})\end{array}$ & $\begin{array}{r}d_{\text {rough }} \\
(\mathrm{nm})\end{array}$ & $S_{1}$ & $\begin{array}{c}\omega_{\mathrm{TO} 1} \\
\left(\mathrm{~cm}^{-1}\right)\end{array}$ & $\begin{array}{c}\gamma_{\mathrm{TO} 1} \\
\left(\mathrm{~cm}^{-1}\right)\end{array}$ & $S_{2}$ & $\begin{array}{c}\omega_{\mathrm{TO} 2} \\
\left(\mathrm{~cm}^{-1}\right)\end{array}$ & $\begin{array}{c}\gamma_{\mathrm{TO} 2} \\
\left(\mathrm{~cm}^{-1}\right)\end{array}$ & $\begin{array}{c}\omega_{\mathrm{P}} \\
\left(\mathrm{cm}^{-1}\right)\end{array}$ & $\begin{array}{c}\gamma_{\mathrm{P}} \\
\left(\mathrm{cm}^{-1}\right)\end{array}$ & $\begin{array}{c}n_{\mathrm{IRSE}} \\
\left(\mathrm{cm}^{-3}\right)\end{array}$ & $\begin{array}{l}m_{\mathrm{opt}}^{*} \\
\left(\mathrm{~m}_{\mathrm{e}}\right)\end{array}$ & $\begin{array}{c}E_{\mathrm{CV}, \mathrm{UVSE}} \\
(\mathrm{eV})\end{array}$ & $\begin{array}{l}E_{\mathrm{CV}} \\
(\mathrm{eV})\end{array}$ \\
\hline A & $3.7 \times 10^{20}$ & 352 & 5.5 & 1.8 & 552 & 8.1 & 2.3 & 543 & 11.7 & 8878 & 390 & $1.78 \times 10^{20}$ & 0.202 & 3.71 & 3.70 \\
\hline B & $8.7 \times 10^{19}$ & 416 & 7.3 & 1.9 & 552 & 7.1 & 2.5 & 544 & 12.2 & 5082 & 419 & $5.58 \times 10^{19}$ & 0.194 & 3.39 & 3.40 \\
\hline $\mathrm{C}$ & $2.7 \times 10^{18}$ & 602 & 4.8 & 2.6 & 552 & 5.2 & 2.4 & 538 & 15.7 & 1039 & 363 & $2.27 \times 10^{18}$ & 0.191 & 3.22 & 3.21 \\
\hline $\mathrm{D}$ & - & 605 & 3.8 & 2.5 & 552 & 5.6 & 1.9 & 547 & 8.2 & - & - & - & 0.189 & 3.23 & 3.23 \\
\hline $\mathrm{E}$ & $3.8 \times 10^{19}$ & 569 & 3.9 & 2.8 & 552 & 7.0 & 2.2 & 545 & 10.1 & 3589 & 488 & $2.75 \times 10^{19}$ & 0.189 & 3.30 & 3.31 \\
\hline $\mathrm{F}$ & $7.6 \times 10^{18}$ & 611 & 8.5 & 2.4 & 552 & 6.7 & 1.9 & 545 & 9.5 & 1921 & 416 & $7.79 \times 10^{18}$ & 0.189 & 3.23 & 3.24 \\
\hline
\end{tabular}

which yield $E_{\mathrm{P}}=13.77 \mathrm{eV}$. Introducing an uncertainty for $m_{0}^{*}$ of $\pm 0.01 \mathrm{~m}_{\mathrm{e}}$ in hindsight, an $E_{\mathrm{P}}$-parameter range between 12.92 and $14.71 \mathrm{eV}$ is derived (Fig. 7). Previous theoretical results suggested a value of $16.86 \mathrm{eV}[54]$ or $\approx 25 \mathrm{eV}[72,73]$ which cannot be confirmed through our analysis. However, the suggested $E_{\mathrm{P}}$ parameters yield band masses of $m_{0}^{*}=0.16 \mathrm{~m}_{\mathrm{e}}$ or $0.11 \mathrm{~m}_{\mathrm{e}}$, respectively, using $E_{\mathrm{G}}=3.23 \mathrm{eV}$, which we consider to be too small for c-GaN. Possibly, the too high value of $E_{\mathrm{P}}$ in Refs. [54], [72], and [73] stems from the implementation of Eq. (7) instead of Eq. (8). Consequently, we recommend to use the value of $E_{\mathrm{P}}=13.77 \mathrm{eV}$ for $\mathrm{c}-\mathrm{GaN}$.

The BGR, included in the calculations of Eqs. (12) and (13) $\left(E_{\mathrm{G}} \rightarrow E_{\text {ren }}\right.$ ), yields a slightly decreasing optical effective mass for increasing carrier concentrations up to $n \approx 2 \times$ $10^{18} \mathrm{~cm}^{-3}$ (Fig. 7). For higher carrier concentrations, the optical effective mass is monotonously increasing.

In the next step, we combine our result for the $E_{\mathrm{P}}$ parameter with the experimentally obtained plasma frequencies of our samples using Eqs. (4) and (13). The result is presented in Fig. 8 as a function of the carrier concentration. We implemented the parameter set $E_{\mathrm{G}}=3.23 \mathrm{eV}, m_{0}^{*}=0.19 \mathrm{~m}_{\mathrm{e}}$, and thus $E_{\mathrm{P}}=13.77 \mathrm{eV}$. It can be seen that the plasma frequency is monotonously increasing as a function of the free-carrier concentration. Therefore, the free-carrier concentration $\left(n_{\mathrm{IRSE}}\right)$ can be determined from the optically obtained $\omega_{\mathrm{P}}$. The resulting values for $n_{\text {IRSE }}$ are given in Table II. These values change less than $\pm 5 \%$ if effective masses $m_{0}^{*}$ between $0.18 \mathrm{~m}_{\mathrm{e}}$ and $0.20 \mathrm{~m}_{\mathrm{e}}$ (and thus $E_{\mathrm{P}}$-parameters between 14.71 and $12.92 \mathrm{eV}$ ) are used.

The optically obtained carrier concentrations $n_{\mathrm{IRSE}}$ deviate slightly from the Hall-effect results $n_{\text {Hall }}$. The highest difference between $n_{\mathrm{IRSE}}$ and $n_{\mathrm{Hall}}$ occurs for sample $\mathrm{A}$ and is about a factor of 2. To obtain the (higher) Hall-effect free-carrier concentration by Eq. (4), the optical effective electron mass has to be twice its actual value since the plasma frequency is validated by two independent measurement techniques (ellipsometry and Raman, please see below). Therefore, we use $n_{\text {IRSE }}$ instead of $n_{\text {Hall }}$ for the further analysis. It can be shown, that there is no possible choice for $E_{\mathrm{P}}$, that describes the value of the free-carrier concentration measured by the Hall
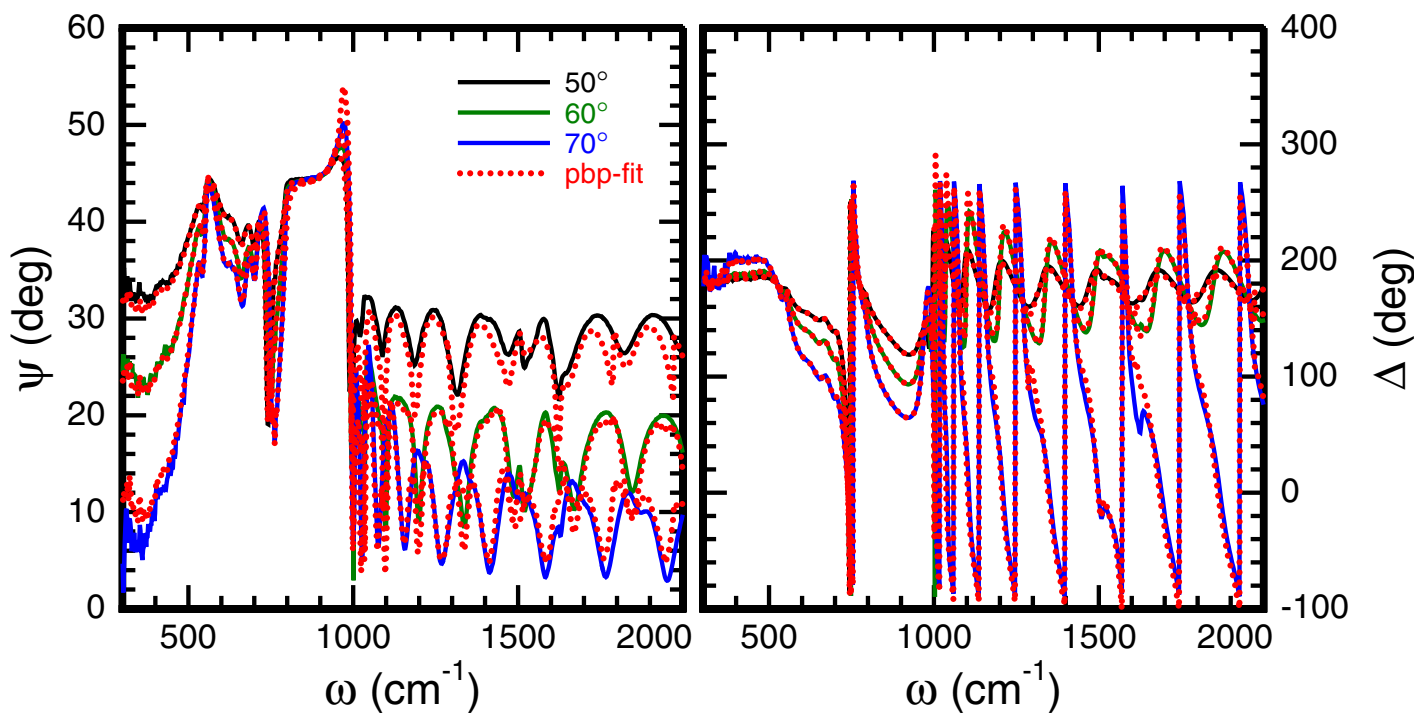

FIG. 4. Ellipsometric parameters $\Psi$ and $\Delta$ of sample D (unintentionally doped) in the infrared range measured by spectroscopic ellipsometry for three angles of incidence (black, green, blue, continuous curves) and the corresponding point-by-point-fit (pbp-fit, red, dotted curves). 


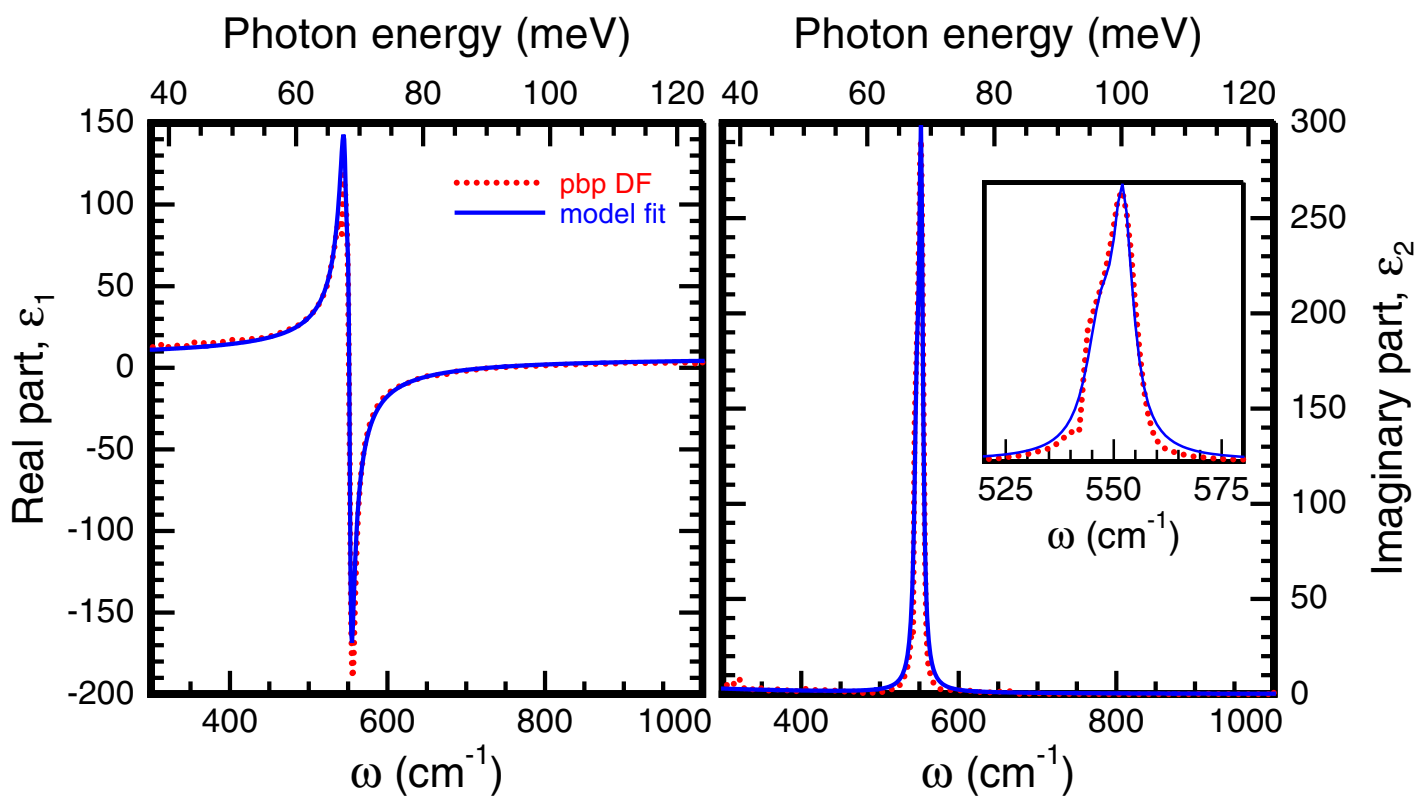

FIG. 5. Point-by-point fitted (pbp DF, dotted curve) and analytical model (model fit, continuous curve) dielectric function for sample D (unintentionally doped) in the infrared range. The inset on the right side displays a magnified spectral region of the phonon contribution. Here, the apparent split of the TO phonon is very distinct.

effect and $\omega_{\mathrm{P}}$ as found by IRSE. A possible explanation of the difference between $n_{\text {IRSE }}$ and $n_{\text {Hall }}$ could be an inaccuracy of the Hall measurements due to the conducting $3 \mathrm{C}-\mathrm{SiC}$ substrate.

Additionally, Raman measurements were performed to support the IRSE measurements. For quantitative comparison of the DFs and Raman spectra, we calculate the imaginary part of the dielectric loss function $\Omega(\omega)$ from pbp-DFs [74]:

$$
\Omega(\omega)=\operatorname{Im}\left(-\frac{1}{\varepsilon(\omega)}\right)=\frac{\varepsilon_{2}}{\varepsilon_{1}^{2}+\varepsilon_{2}^{2}} .
$$

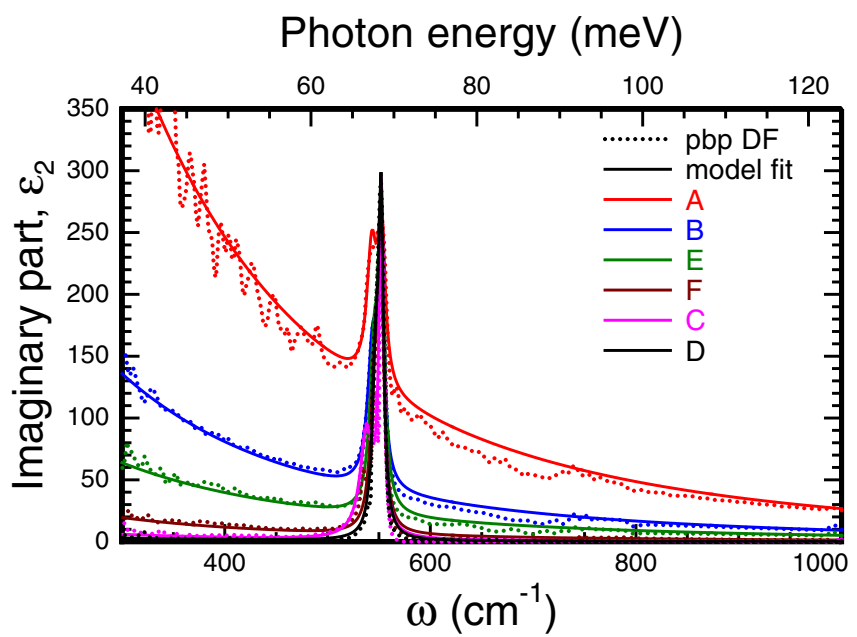

FIG. 6. Point-by-point fitted (pbp DF, dotted curves) imaginary parts of the dielectric functions of the five doped samples and the reference sample as well as the corresponding analytical line shape fits (model fit, continuous curves).
In the range $\omega<1000 \mathrm{~cm}^{-1}$, the Raman spectra are dominated by phonons from the substrates, namely the $\mathrm{TO}(\Gamma)_{\mathrm{Si}}$ at $521 \mathrm{~cm}^{-1}$, the $\operatorname{TO}(\Gamma)_{3 \mathrm{C}-\mathrm{SiC}}$ at $795 \mathrm{~cm}^{-1}$, and the $\operatorname{LO}(\Gamma)_{\mathrm{Si}}$ at $972 \mathrm{~cm}^{-1}$ [75]. The $\mathrm{TO}(\Gamma)_{\mathrm{c}-\mathrm{GaN}}$ phonon mode is forbidden and therefore not visible in these Raman spectra [76,77]. At higher wave numbers $\left(\omega \geqslant 2000 \mathrm{~cm}^{-1}\right) \Omega(\omega)$ expresses a broad peak at exactly the same position where a weak Raman signal is found (Fig. 9). The loss function peaks at

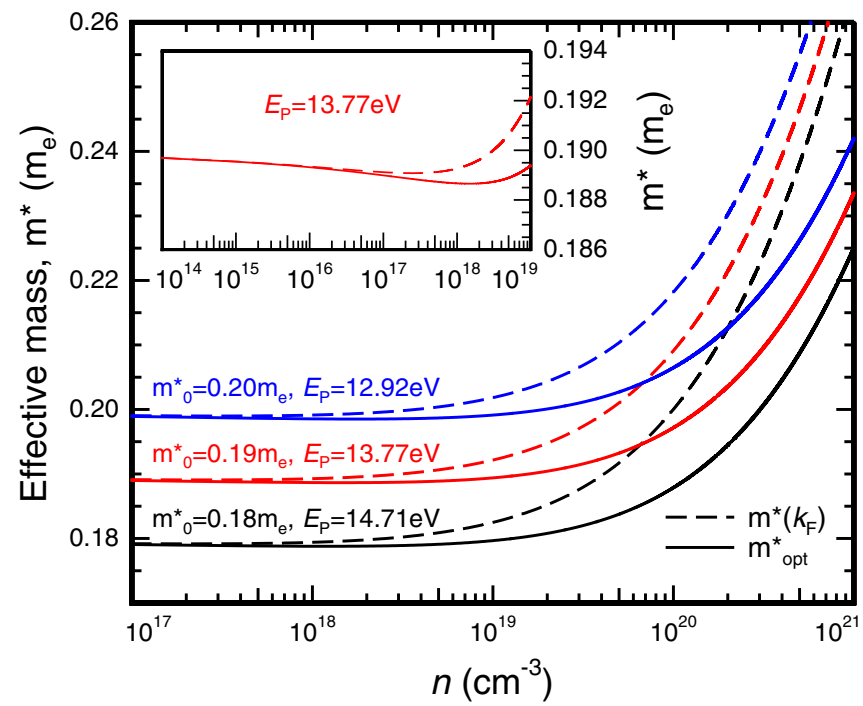

FIG. 7. Effective mass at the Fermi vector $\left[m^{*}\left(k_{\mathrm{F}}\right)\right.$, dashed curves] and optical effective mass ( $m_{\mathrm{opt}}^{*}$, continuous curves) calculated by Eq. (12) as a function of free-carrier concentration $n$. Bandgap renormalization effects induce a decreasing optical effective mass until $n \approx 2 \times 10^{18} \mathrm{~cm}^{-3}$ (see inset). The effective mass at the $\Gamma$-point $m_{0}^{*}$ determines $E_{\mathrm{P}}$. $\Gamma$-point masses between $0.18-0.20 \mathrm{~m}_{\mathrm{e}}$ yield values for $E_{\mathrm{p}}$ between $14.71-12.92 \mathrm{eV}$. 


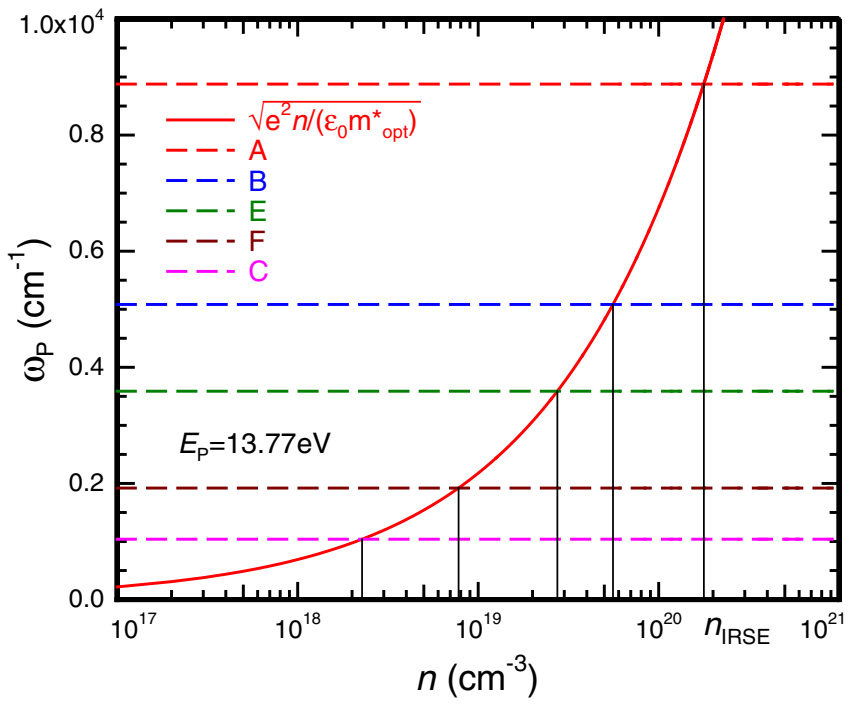

FIG. 8. Plasma frequency $\omega_{\mathrm{P}}$ calculated by Eq. (12) (red, continuous curve) including band-gap renormalization effects and experimentally obtained plasma frequencies for different samples (dashed lines). The intersections of these lines determine the free-carrier concentrations $n_{\text {IRSE }}$, which are slightly different from $n_{\text {Hall }}$.

$\varepsilon_{1} \rightarrow 0$. In a classic picture, this Raman signal is identified with the longitudinal optical phonon-plasmon coupled $(\mathrm{LPP}+)$ mode [78,79]. The position of the LPP + mode is characteristic for the plasma frequency of the sample, which therefore acts as an independent validation of the measured plasma frequency. Please note, that the calculation of the LPP + modes considers the screened plasma frequency $\omega_{\mathrm{P}}^{*}=\omega_{\mathrm{P}} / \sqrt{\varepsilon_{\infty}}$.

In our case, we are able to confirm the accuracy of the determined DFs and thus the values for $\omega_{\mathrm{P}}$. While the position and width of the Raman peak is in very good agreement with the dielectric loss spectrum of the two highest doped samples, the same comparison cannot be easily made for the lower

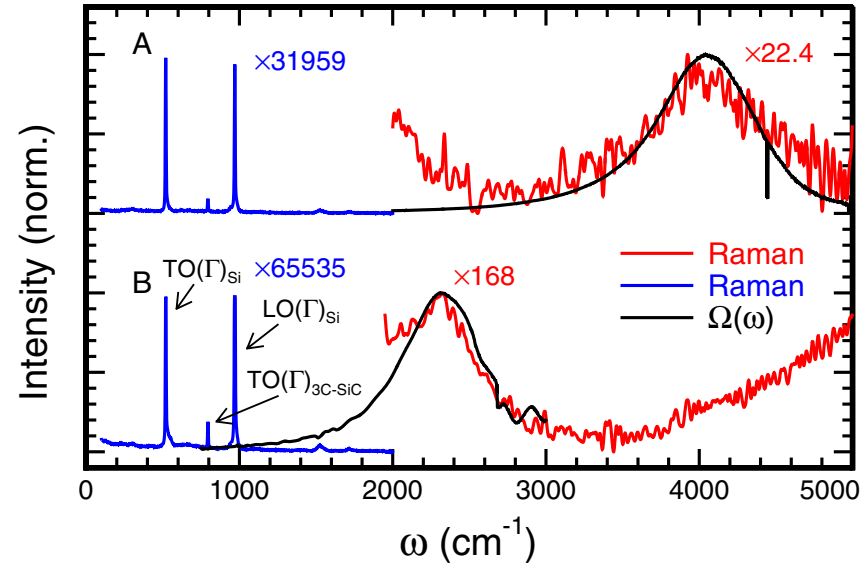

FIG. 9. Normalized dielectric loss spectra [ $\Omega(\omega)$, black] of samples $\mathrm{A}\left(n_{\text {Hall }}=3.7 \times 10^{20} \mathrm{~cm}^{-3}\right)$ and $\mathrm{B}\left(n_{\text {Hall }}=8.7 \times 10^{19} \mathrm{~cm}^{-3}\right)$ compared to Raman spectra (blue, red). For clarity, the spectra are normalized to the maximum of each curve and shifted vertically. The Raman spectra were normalized separately for the ranges below and above $2000 \mathrm{~cm}^{-1}$.

doped samples due to overlapping higher-order Raman bands and substrate modes.

\section{B. Ultraviolet spectroscopic ellipsometry}

Similar to the analysis in the infrared region, all samples were measured in the visible and ultra-violet spectral range by spectroscopic ellipsometry, as described in Sec. II. However, here, another layer was added to the multilayer stack model to account for a surface roughness on top of the c-GaN layer. The surface roughness was described by a Bruggeman effective medium approximation (50\% void in $\mathrm{c}-\mathrm{GaN}$ matrix), which determines an effective DF from the two components [80]. The thickness of the surface roughness $d_{\text {rough }}$ for the different samples can be found in Table II. Again, $\Psi$ and $\Delta$ are fitted point-by-point using a multilayer model. The excellent

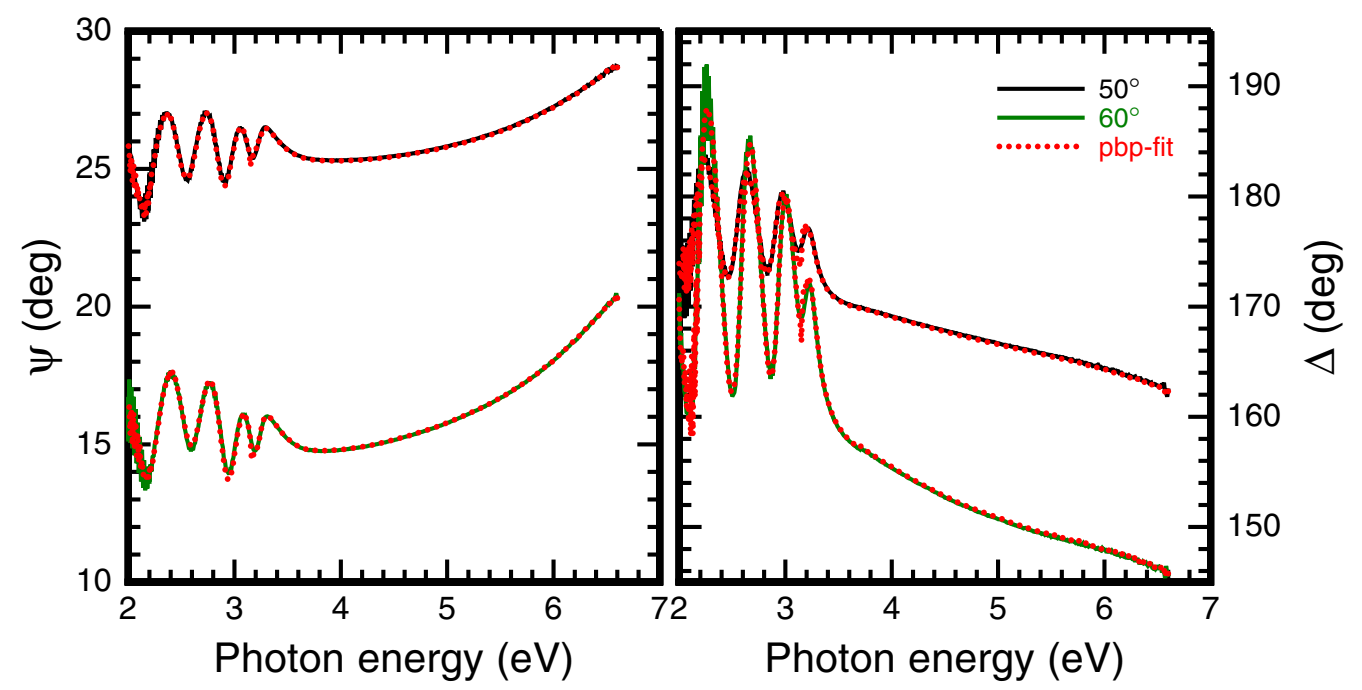

FIG. 10. Ellipsometric parameters $\Psi$ and $\Delta$ of sample $\mathrm{E}\left(n_{\text {Hall }}=3.8 \times 10^{19} \mathrm{~cm}^{-3}\right)$ in the visible and ultraviolet range measured by spectroscopic ellipsometry for two angles of incidence (black, green, continuous curves) and the corresponding point-by-point-fit (pbp-fit, red, dotted curves). 


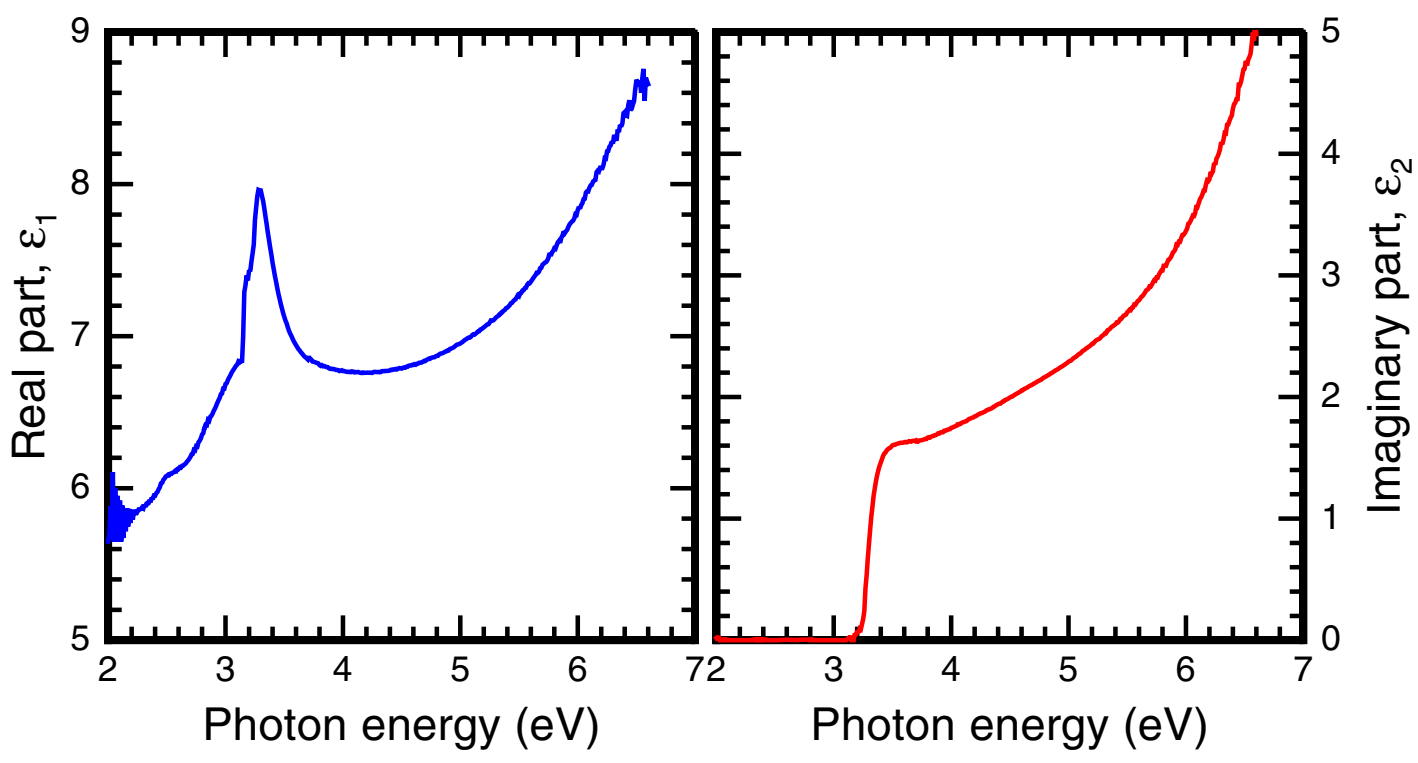

FIG. 11. Point-by-point fitted dielectric function for sample $\mathrm{E}\left(n_{\mathrm{Hall}}=3.8 \times 10^{19} \mathrm{~cm}^{-3}\right)$ in the visible and ultraviolet range.

agreement with the experimental data can be seen in Fig. 10 for the example of sample E. This was performed for all samples. In the lower photon energy region, Fabry-Pérot oscillations are visible which vanish at the absorption edge. These oscillations are predominant in determining the layer thicknesses $d_{\mathrm{UVSE}}$ of the c-GaN layer and $d_{\text {rough }}$ of the surface roughness tabulated in Table II as well as the thicknesses of the substrate layers.

The determined real and imaginary parts of the pbp-DF are shown in Fig. 11 for the same sample. The energy position of the absorption edge $E_{\mathrm{CV} \text {, UVSE }}$ was determined by analysis of the inflection point in the imaginary part of the pbp-DF $\varepsilon_{2}$ since the line shape around the absorption edge basically resembles a Fermi-Dirac distribution and thus yields the Fermi-vector. The interband transition energy of sample E was determined to be $3.30 \mathrm{eV}$. Please note that irregularities in $\varepsilon_{1}$ for photon energies below $3.28 \mathrm{eV}$ are residuals from Fabry-Pérot oscillations of the multilayer stack.

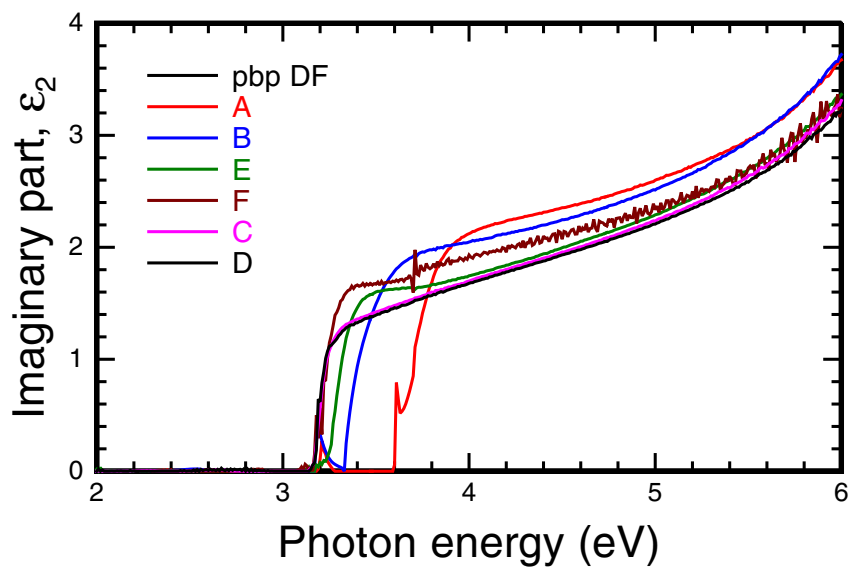

FIG. 12. Point-by-point data for the imaginary part of the dielectric functions (pbp DF) of all samples as experimentally determined by spectroscopic ellipsometry in the visible and ultraviolet range.
The imaginary parts of the pbp-DFs of all samples are shown in Fig. 12. Obviously, the absorption edge blue-shifts for an increasing free-carrier concentration similar to the case of wurtzite $\mathrm{GaN}$ [41]. In our case, $E_{\mathrm{CV} \text {,UVSE }}$ varies from $\approx 3.22 \mathrm{eV}$ (sample $\mathrm{C}$ ) to $\approx 3.71 \mathrm{eV}$ (sample A).

The effects of BMS and BGR on $E_{\mathrm{CV}}$ as a function of the free-carrier concentration are described by Eqs. (15) and (19) using an averaged hole mass as given in Eq. (20). The analytical description of $E_{\mathrm{CV}}$ as a function of the free-carrier concentration to the experimental results $E_{\mathrm{CV} \text {,UVSE }}$ is shown in Fig. 13. There, the contributions of BMS and BGR are, in addition, plotted separately to make the magnitudes of both influences more apparent. By varying $\bar{m}_{\mathrm{h}}$ and therefore the

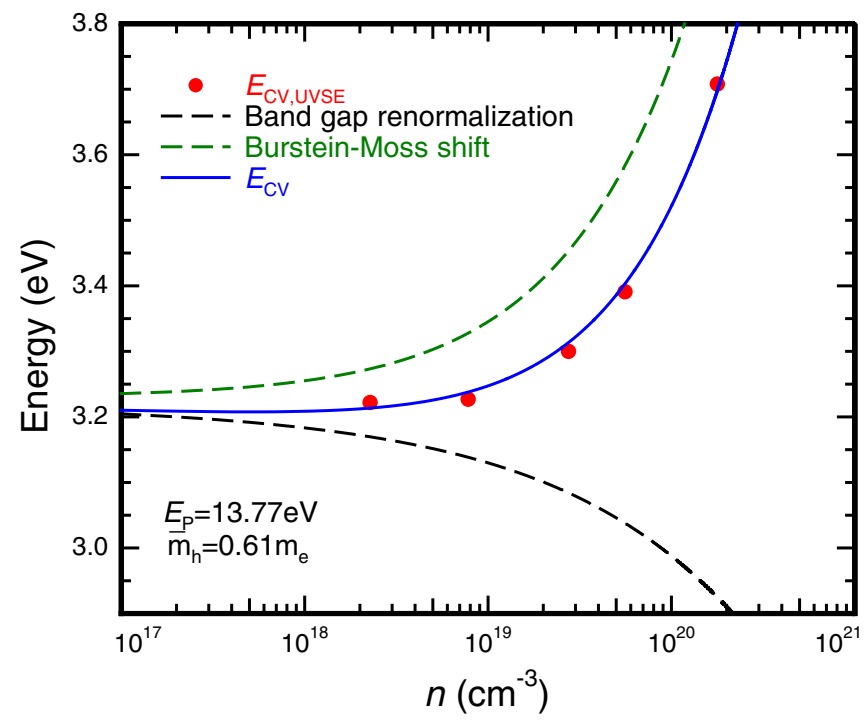

FIG. 13. Experimentally obtained transition energies ( $E_{\mathrm{CV}, \mathrm{UVSE}}$, symbols) compared to the analytical description $\left(E_{\mathrm{CV}}\right.$, continuous curve). The contributions of band-gap renormalization (black, dashed curve) and Burstein-Moss shift (green, dashed curve) are shown separately. 
BMS contribution, we are able to fit the analytical description of the transition energy to the experimental data. A very good agreement between the model description of BMS and BGR in combination with the nonparabolic band structure model (Sec. IIIB) and the experimental data is achieved with an averaged but constant hole mass of $\bar{m}_{\mathrm{h}}=0.61 \mathrm{~m}_{\mathrm{e}}$. It should be noted that similar good agreements can be obtained using other values for $E_{\mathrm{P}}$ within the range $12.92-14.71 \mathrm{eV}$, corresponding to band masses between $0.18-0.20 \mathrm{~m}_{\mathrm{e}} \cdot \bar{m}_{\mathrm{h}}$ would then be found between $0.73 \mathrm{~m}_{\mathrm{e}}$ (for $m_{0}^{*}=0.18 \mathrm{~m}_{\mathrm{e}}$ ) and $0.56 \mathrm{~m}_{\mathrm{e}}$ (for $m_{0}^{*}=0.20 \mathrm{~m}_{\mathrm{e}}$ ). However, we remind the reader that the determination of $E_{\mathrm{P}}$ is completely independent of $\bar{m}_{\mathrm{h}}$.

\section{SUMMARY}

In conclusion, the optical and plasmonic properties of zincblende $\mathrm{GaN}$, grown by $\mathrm{MBE}$ on a $3 \mathrm{C}-\mathrm{SiC} / \mathrm{Si}$ substrate and doped by $\mathrm{Ge}$ and $\mathrm{Si}$ up to $n>10^{20} \mathrm{~cm}^{-3}$, were investigated by spectroscopic ellipsometry. Additionally, Raman measurements were performed. An excellent agreement between the measured spectra and the applied model was achieved. The DF in the infrared and visible spectral range, obtained by spectroscopic ellipsometry, yields a phonon and a plasmon contribution and determines the transition energy. A model containing two TO phonons was necessary, in line with previous investigations. Furthermore, we presented a selfconsistent, all-optical approach to determine the free-carrier concentration and band parameters, like the $E_{\mathrm{P}}$ parameter and the optical effective electron mass, by using Kane's model as well as the plasma frequency and the transition energy. Effects of BGR and BMS were observed and included into the theoretical approach. Therefore, the effective electron masses were determined. An excellent agreement between the increasing interband transition energy and the applied model was achieved and an averaged constant effective hole mass was determined.

\section{ACKNOWLEDGMENTS}

We gratefully acknowledge support by the Deutsche Forschungsgemeinschaft in the framework of Major Research Instrumentation Programs No. INST 272/230-1 and via project B02 within the Transregio program TRR 142 Project No. 231447078.
[1] L. Shunfeng, J. Schörmann, D. J. As, and K. Lischka, Appl. Phys. Lett. 90, 071903 (2007).

[2] M. T. Durniak, A. S. Bross, D. Elsaesser, A. Chaudhuri, M. L. Smith, A. A. Allerman, S. C. Lee, R. J. Brueck, and C. Wetzel, Adv. Electron. Mater. 2, 1500327 (2016).

[3] B. M. Shi, M. H. Xi, H. S. Wu, N. Wang, and S. Y. Tong, Appl. Phys. Lett. 89, 151921 (2006).

[4] R. M. Kemper, L. Hiller, T. Stauden, J. Pezoldt, K. Duschik, T. Niendorf, H. J. Maier, D. Meertens, K. Tillmann, D. J. As, and J. K. N. Lindner, J. Cryst. Growth 378, 291 (2013).

[5] B. Kunert, Y. Mols, M. Baryshniskova, N. Waldron, A. Schulze, and R. Langer, Semicond. Sci. Technol. 33, 093002 (2018).

[6] L. Y. Lee, Mater. Sci. Technol. 33, 1570 (2017).

[7] R. Liu, R. Schaller, C. Q. Chen, and C. Bayram, ACS Photon. 5, 955 (2018).

[8] L. Y. Lee, M. Frentrup, M. J. Kappers, R. A. Oliver, C. J. Humphreys, and D. J. Wallis, J. Appl. Phys. 124, 105302 (2018).

[9] S. Kako, M. Miyamura, K. Tachibana, K. Hoshino, and Y. Arakawa, Appl. Phys. Lett. 83, 984 (2003).

[10] M. Bürger, T. Schupp, K. Lischka, and D. J. As, Phys. Status Solidi C 9, 1273 (2012).

[11] M. Bürger, M. Ruth, S. Declair, J. Förstner, C. Meier, and D. J. As, Appl. Phys. Lett. 102, 081105 (2013).

[12] C. J. M. Stark, T. Detchprohm, S. C. Lee, Y.-B. Jiang, S. R. J. Brueck, and C. Wetzel, Appl. Phys. Lett. 103, 232107 (2013).

[13] S. Sergent, S. Kako, M. Bürger, T. Schupp, D. J. As, and Y. Arakawa, Phys. Rev. B 90, 235312 (2014).

[14] S. Kako, M. Holmes, S. Sergent, M. Bürger, D. J. As, and Y. Arakawa, Appl. Phys. Lett. 104, 011101 (2014).

[15] M. Bürger, J. K. N. Lindner, D. Reuter, and D. J. As, Phys. Status Solidi C 12, 452 (2015).

[16] T. Wecker, F. Hörich, M. Feneberg, R. Goldhahn, D. Reuter, and D. J. As, Phys. Status Solidi B 252, 873 (2015).
[17] S. Sergent, S. Kako, M. Bürger, S. Blumenthal, S. Iwamoto, D. J. As, and Y. Arakawa, Appl. Phys. Express 9, 012002 (2016).

[18] T. Wecker, G. Callsen, A. Hoffmann, D. Reuter, and D. J. As, Jpn. J. Appl. Phys. 55, 05FG01 (2016).

[19] S. Blumenthal, D. Reuter, and D. J. As, Phys. Status Solidi B 255, 1700457 (2018).

[20] M. Feneberg, M. Winkler, K. Lange, M. Wieneke, H. Witte, A. Dadgar, and R. Goldhahn, Appl. Phys. Express 11, 101001 (2018).

[21] T. Hiroshima and R. Lang, Appl. Phys. Lett. 49, 456 (1986).

[22] C. Wetzel, R. Winkler, M. Drechsler, B. K. Meyer, U. Rössler, J. Scriba, J. P. Kotthaus, V. Härle, and F. Scholz, Phys. Rev. B 53, 1038 (1996).

[23] L. D. Whalley, J. M. Frost, B. J. Morgan, and A. Walsh, Phys. Rev. B 99, 085207 (2019).

[24] M. Deppe, J. W. Gerlach, D. Reuter, and D. J. As, Phys. Status Solidi B 254, 1600700 (2017).

[25] M. Deppe, J. W. Gerlach, S. Shvarkov, D. Gogalla, H.-W. Becker, D. Reuter, and D. J. As, J. Appl. Phys. 125, 095703 (2019).

[26] S. Fritze, A. Dadgar, H. Witte, M. Bügler, A. Rohrbeck, J. Bläsing, A. Hoffmann, and A. Krost, Appl. Phys. Lett. 100, 122104 (2012).

[27] M. Wieneke, H. Witte, K. Lange, M. Feneberg, A. Dadgar, J. Bläsing, R. Goldhahn, and A. Krost, Appl. Phys. Lett. 103, 012103 (2013).

[28] R. Kirste, M. P. Hoffmann, E. Sachet, M. Bobea, Z. Bryan, I. Bryan, C. Nenstiel, A. Hoffmann, J.-P. Maria, R. Collazo, and Z. Sitar, Appl. Phys. Lett. 103, 242107 (2013).

[29] A. Ajay, J. Schörmann, M. Jiménez-Rodriguez, C. B. Lim, F. Walther, M. Rohnke, I. Mouton, L. Amichi, C. Bougerol, M. I. Den Hertog, M. Eickhoff, and E. Monroy, J. Phys. D 49, 445301 (2016). 
[30] E. O. Kane, J. Phys. Chem. Solids 1, 249 (1957).

[31] T. S. Moss, Proc. Phys. Soc. B 67, 775 (1954).

[32] E. Burstein, Phys. Rev. 93, 632 (1954).

[33] K.-F. Berggren and B. E. Sernelius, Phys. Rev. B 24, 1971 (1981).

[34] D. J. As, A. Richter, J. Busch, B. Schöttker, M. Lübbers, J. Mimkes, D. Schikora, K. Lischka, W. Kriegseis, W. Burkhardt, and B. K. Meyer, MRS Internet J. Nitride Semicond. Res. 5, 308 (2000)

[35] D. J. As, Defect Diffus. Forum 206, 87 (2002).

[36] D. J. As, M. Deppe, J. Gerlach, and D. Reuter, MRS Adv. 2, 283 (2017).

[37] S. Shokhovets, R. Goldhahn, G. Gobsch, S. Piekh, R. Lantier, A. Rizzi, V. Lebedev, and W. Richter, J. Appl. Phys. 94, 307 (2003).

[38] D. Schmidt and M. Schubert, J. Appl. Phys. 114, 083510 (2013).

[39] M.-Y. Xie, M. Schubert, J. Lu, P. O. A. Persson, V. Stanishev, C. L. Hsiao, L. C. Chen, W. J. Schaff, and V. Darakchieva, Phys. Rev. B 90, 195306 (2014).

[40] R. Goldhahn, Acta Phys. Pol. A 104, 123 (2003).

[41] M. Feneberg, S. Osterburg, K. Lange, C. Lidig, B. Garke, R. Goldhahn, E. Richter, C. Netzel, M. D. Neumann, N. Esser, S. Fritze, H. Witte, J. Bläsing, A. Dadgar, and A. Krost, Phys. Rev. B 90, 075203 (2014).

[42] M. Feneberg, J. Nixdorf, C. Lidig, R. Goldhahn, Z. Galazka, O. Bierwagen, and J. S. Speck, Phys. Rev. B 93, 045203 (2016).

[43] J.-M. Costantini, S. Miro, and O. Pluchery, J. Phys. D: Appl. Phys. 50, 095301 (2017).

[44] Ch. Zgheib, Ch. Förster, P. Weih, V. Cimalla, M. Kazan, P. Masri, O. Ambacher, and J. Pezoldt, Thin Solid Films 455, 183 (2004).

[45] J. Pezoldt, Ch. Zgheib, V. Lebedev, P. Masri, and O. Ambacher, Supperlattices and Microstructures 40, 612 (2006).

[46] T. Werninghaus, M. Friedrich, V. Cimalla, J. Scheiner, R. Goldhahn, D. R. T. Zahn, and J. Pezoldt, Diam. Relat. Mater. 7, 1385 (1998).

[47] J. Scheiner, R. Goldhahn, V. Cimalla, G. Ecke, W. Attenberger, J. K. M. Lindner, G. Gobsch, and J. Petzoldt, Mater. Sci. Eng. B 61-62, 526 (1999).

[48] F. Wooten, Optical Properties of Solids (Acadamic Press, New York, 1972), p. 42.

[49] M. Feneberg, M. Röppischer, C. Cobet, N. Esser, J. Schörmann, T. Schupp, D. J. As, F. Hörich, J. Bläsing, A. Krost, and R. Goldhahn, Phys. Rev. B 85, 155207 (2012).

[50] P. Y. Yu and M. Cardona, Fundamentals of Semiconductors: Physics and Materials Properties, 4th ed. (Springer, Berlin, 2010), p. 260.

[51] G. Ramirez-Flores, H. Navarro-Contreras, A. Lastras-Martínez, R. C. Powell, and J. E. Greene, Phys. Rev. B 50, 8433 (1994).

[52] T. Lei, T. D. Moustakas, R. J. Graham, Y. He, and S. J. Berkowitz, J. Appl. Phys. 71, 4933 (1992).

[53] J. Wu, W. Walukiewicz, W. Shan, K. M. Yu, J. W. Ager, E. E. Haller, H. Lu, and W. J. Schaff, Phys. Rev. B 66, 201403(R) (2002).

[54] P. Rinke, M. Winkelnkemper, A. Qteish, D. Bimberg, J. Neugebauer, and M. Scheffler, Phys. Rev. B 77, 075202 (2008).
[55] C. Persson, A. Ferreira da Silva, R. Ahuja, and B. Johansson, J. Cryst. Growth 231, 397 (2001).

[56] L. C. de Carvalho, A. Schleife, and F. Bechstedt, Phys. Rev. B 84, 195105 (2011)

[57] P. Schley, R. Goldhahn, C. Napierala, G. Gobsch, J. Schörmann, D. J. As, K. Lischka, M. Feneberg, and K. Thonke, Semicond. Sci. Technol. 23, 055001 (2001).

[58] M. Feneberg, K. Lange, C. Lidig, M. Wieneke, H. Witte, J. Bläsing, A. Dadgar, A. Krost, and R. Goldhahn, Appl. Phys. Lett. 103, 232104 (2013).

[59] P. Kühne, T. Hoffmann, M. Schubert, D. Nilsson, A Kakanakova-Georgieve, E. Janzén, and V. Darakchieva, Appl. Phys. Lett. 103, 212107 (2013).

[60] W. G. Spitzer and H. Y. Fan, Phys. Rev. 106, 882 (1957).

[61] M. P. Hasselbeck and P. M. Enders, Phys. Rev. B 57, 9674 (1998).

[62] P. Schley, R. Goldhahn, A. T. Winzer, G. Gobsch, V. Cimalla, O. Ambacher, H. Lu, W. J. Schaff, M. Kurouchi, Y. Nanishi, M. Rakel, C. Cobet, and N. Esser, Phys. Rev. B 75, 205204 (2007).

[63] F. Ruske, A. Pflug, V. Sittinger, B. Szyszka, D. Greiner, and B. Rech, Thin Solid Films 518, 1289 (2009).

[64] R. A. Abram, G. J. Rees, and B. L. H. Wilson, Adv. Phys. 27, 799 (1978).

[65] J. M. Luttinger and W. Kohn, Phys. Rev. 97, 869 (1955).

[66] G. Mirjalili, T. J. Parker, S. F. Shayesteh, M. M. Bülbül, S. R. P. Smith, T. S. Cheng, and C. T. Foxon, Phys. Rev. B 57, 4656 (1998).

[67] A. Kasic, M. Schubert, T. Frey, U. Köhler, D. J. As, and C. M. Herzinger, Phys. Rev. B 65, 184302 (2002).

[68] S. C. Lee, S. S. Ng, H. Abu Hassan, Z. Hassan, N. Zainal, S. V. Novikov, C. T. Foxon, and A. J. Kent, Mater. Chem. Phys. 146, 121 (2014).

[69] R. Cuscó, N. Domènech-Amador, S. Novikov, C. T. Foxon, and L. Artús, Phys. Rev. B 92, 075206 (2015).

[70] S. V. Novikov, N. Zainal, A. V. Akimov, C. R. Staddon, A. J. Kent, and C. T. Foxon, J. Vac. Sci. Technol. B 28, C3B1 (2010).

[71] K. Kim, W. R. L. Lambrecht, B. Segall, and M. van Schilfgaarde, Phys. Rev. B 56, 7363 (1997).

[72] A. T. Meney, E. P. O'Reilly, and A. R. Adams, Semicond. Sci. Technol. 11, 897 (1996)

[73] S. K. Pugh, D. J. Dugdale, S. Brand, and R. A. Abram, Semicond. Sci. Technol. 14, 23 (1999).

[74] N. B. Sedrine, A. Zukauskaite, J. Birch, J. Jensen, L. Hultman, S. Schoeche, M. Schubert, and V. Darakchieva, J. Phys. D: Appl. Phys. 48, 415102 (2015).

[75] M. Rüsing, T. Wecker, G. Berth, D. J. As, and A. Zrenner, Phys. Status Solidi B 253, 778 (2016).

[76] A. Tabata, R. Enderlein, J. R. Leite, S. W. da Silva, J. C. Galzernani, D. Schikora, M. Kliodt, and K. Lischka, J. Appl. Phys. 79, 4137 (1996).

[77] C. Hamaguchi, Basic Semiconductor Physics, 2nd ed. (Springer, Berlin, 2010), p. 169.

[78] R. Cuscó, L. Artús, S. Hernández, J. Ibáñez, and M. Hopkinson, Phys. Rev. B 65, 035210 (2001).

[79] Yu. A. Pusep, M. T. O. Silva, J. R. L. Fernandez, V. A. Chitta, J. R. Leite, T. Frey, D. J. As, D. Schikora, and K. Lischka, J. Appl. Phys. 91, 6197 (2002)

[80] D. A. G. Bruggeman, Ann. Phys. (NY) 416, 636 (1935). 